\title{
Role of MAP2K6 in sensitivity to radiotherapy of human nasopharyngeal carcinoma CNE cells.
}

\author{
Jun Fu, Zhanzhan Li*, Na Li \\ Department of Oncology, Xiangya Hospital, Central South University, Changsha, Hunan Province 410008, PR China
}

\begin{abstract}
Background: Radiation therapy is one of the main treatment of nasopharyngeal carcinoma. This study explored the influence of mitogen-activated protein kinase kinase 6 (MAP2K6) on nasopharyngeal carcinoma radiotherapy sensitivity and its possible mechanism.

Methods: Human NPC sensitivity and resistant cell line CNE multicellular spheroids (MCS) were constructed with three dimensional cell culture methods. Cell counting using blood cell counter was employed to detect the radio-sensitivity of CNE MCS before and after blocking MAP2K6 function; the MAP2K6 expression of CNE MCS and cell apoptosis was detected by flow cytometry.

Results: The expression of MAP2K6 increased by elevation of irradiation dose. 0Gy: $12.8 \pm 1.40 .20$, $0.5 \mathrm{~Gy}: 17.6 \pm 2.5 ; 1.0 \mathrm{~Gy}: 22.9 \pm 2.3$; $1.5 \mathrm{~Gy}: 28.3 \pm 2.8$; $2.0 \mathrm{~Gy}: 33.2 \pm 2.9$. There was significantly difference in between blocking group and unblocking group (2Gy: 81\% vs. 65.8\%; 4Gy: 69.2\% vs. 48.3\%; 6Gy: 35.6\% vs. $20.4 \%$; 8Gy: $10.3 \%$ vs. $6.8 \%$.). The apoptosis rate did not significantly increase after $2 \mathrm{~Gy}$ irradiation $(\mathrm{P}>\mathbf{0 . 0 5})$ before being blocked. The $\mathrm{CNE}$ cell significantly upregulated under the 2 Gy irradiation when the expression MAP2K6 was blocked $(\mathrm{P}<0.05)$.

Conclusion: The high expression of MAP2K6 was associated with radio resistance in human NPC CNE cells. MAP2K6 may a potential biomarker of NPC radio sensitivity. Further research was required.
\end{abstract}

Keywords: Nasopharyngeal carcinoma, Radiotherapy, Mitogen-activated protein kinase kinase 6, MAP2K6.

Accepted on December 30, 2017

\section{Introduction}

Nasopharyngeal carcinoma (NPC) is an Epstein-Barr virusassociated epithelial malignancy, with unusual disparity in ethnic and geographical distributions [1]. NPC is a typical regional disease, and most of patients with NPC appeared in South China and Southeast Asia areas [2]. The incidence ranges from $30 \%$ ten thousand to $50 \%$ ten thousand [3]. The incidence and morality of NPC ranked first around the world. NPC has become an important public health issue in these areas. NPC has high malignant degree, and prone to have lymph node metastasis and distant metastasis without obvious symptoms at early stage. Patients have been in the middle and late stage when being confirmed [4]. The radiotherapy is the primary treatment method. In recent decades, the application of intensity modulated radiation therapy improved the curative effect of radiotherapy for NPC with the great development of radio technology and involvement of electronic computer [5]. Most patients can benefit a lot from the radiotherapy. It was reported that the five-year survival rate of patients with I-IVB stage NPC was approximately $85 \%$ [6]. However, there were still some patients who cannot benefit from radiotherapy. The rates of local recurrence and distant metastasis were $8.2 \%$ and $20 \%$, respectively [7]. The local recurrence and metastasis caused by radioresistance become a bottleneck of curative effect for patients with NPC [8,9]. As a complex heterogeneous group, the tumor has extensive information interaction with the surrounding environment [10-12]. Most tumor cells can form cell clusters (multicellular spheroids, MCS) under the condition of three-dimensional, its biological characteristics and the responses to the rays and cytotoxic drugs were significantly different from ordinary monolayer culture cells, but was close to cells in the body. Radiation therapy is one of the main treatment of nasopharyngeal carcinoma. The mitogen activated protein kinase (MAPK) includes extracellular regulated protein kinases, C-JUN Nterminal kinase, and p38 and ERK5 protein kinase. These proteins were important signal molecule. MAP3K6 was upstream kinase of p38 that was involved in cell proliferation, development and division. As a king of extracellular signalregulated kinase, MAP2K was the integration points of multiple biochemical signaling pathways. This study explored the influence of mitogen-activated protein kinase kinase 6 (MAP2K6) on nasopharyngeal carcinoma radiotherapy sensitivity and its possible mechanism.

\section{Materials and Methods}

\section{Establishment of NPC radioresistant cell}

Human CNE cells were from Cancer institute of Xiangya Hospital. Generally, we firstly used the sublethal radiation does 
in NPC CNE-2 line. Almost all NPC cells were killed after five days of irradiation at $12 \mathrm{~Gy}$. Then we used a dose of $11 \mathrm{~Gy}$ for sublethal for selecting CNE2 radioresistant cells. The first generation of subclone cells was generated from the surviving fraction of the parental cells irradiated with the sublethal dose. We repeated this process until the fifth generation CNE cells was considered as the CNE2 cell radio resistant group.

\section{Protein extraction}

Sample was sonicated three times on ice using a high intensity ultrasonic processor (Scientz) in lysis buffer ( $8 \mathrm{M}$ urea, $2 \mathrm{mM}$ EDTA, $10 \mathrm{mM}$ DTT, 1\% Phosphatase Inhibitor Cocktail and $1 \%$ Protease Inhibitor Cocktail). The remaining debris was removed by centrifugation at $20,000 \mathrm{~g}$ at $4{ }^{\circ} \mathrm{C}$ for $10 \mathrm{~min}$. Finally, the protein was precipitated with cold $15 \%$ TCA for 2 $\mathrm{h}$ at $-20^{\circ} \mathrm{C}$. After centrifugation at $4^{\circ} \mathrm{C}$ for $10 \mathrm{~min}$, the supernatant was discarded. The remaining precipitate was washed with cold acetone for three times. The protein was dissolved in buffer ( $8 \mathrm{M}$ urea, $100 \mathrm{mM}$ TEAB, and $\mathrm{pH}$ 8.0) and the protein concentration was determined with 2-D Quant kit according to the manufacturer's instructions.

\section{Examination of radio sensitivity and apoptosis}

We used MAP2K6 antibody $(5 \mathrm{mug} / \mathrm{ml})$ to block the expression of MAP2K6. Each group received 0, 2, 4, 6, 8 Gy irradiation dose continue to cultivate $48 \mathrm{~h}$, digestion process of preparation of single cell suspension, centrifugal, abandon supernatant, adding $0.01 \mathrm{~m}$ PBS beat into single cell suspension, absorbing amount of single cell suspension in blood cell counter, counting the microscopic cell, drawing dose and cell activity curve. Cell survival rate=number of survival cells in different doses of irradiation group/unexposed group* $100 \%$. Apoptosis was examined by flow cytometry. Single cell suspension of Control group, radiation group and MAP2K6 blocking group (cultivating for $24 \mathrm{~h}$ ) was made for flow cytometry examination.

\section{Statistical analysis}

We used SPSS 14.0 to perform the statistical analyses. The continuous data was expressed by using mean \pm standard deviation. We used t-test to compare the difference between groups.

\section{Results}

\section{Expression of MAP2K6}

After 5-day culture, we can saw a three-dimensional structure CNE MCS with the diameter of $100 \mathrm{~mm}$. The expression of MAP2K6 increased by elevation of irradiation dose. 0Gy: 12.8 \pm 1.40 .20 , 0.5Gy: $17.6 \pm 2.5$; $1.0 \mathrm{~Gy}: 22.9 \pm 2.3$; $1.5 \mathrm{~Gy}: 28.3 \pm$ 2.8; $2.0 \mathrm{~Gy}: 33.2 \pm 2.9$.

\section{Cell survival rate}

We used the cell survival rate to compare the radio sensitivity. The higher cell survival rate means lower radio sensitivity. The cell survival rate of blocking and unblocking group were $2 \mathrm{~Gy}$ : 81\% vs. $65.8 \%$; 4Gy: $69.2 \%$ vs. $48.3 \%$; 6Gy: $35.6 \%$ vs. $20.4 \%$; 8Gy: $10.3 \%$ vs. $6.8 \%$. There was significantly difference in between blocking group and unblocking group $(\mathrm{P}<0.05)$. The Figure 1 presented the cell survival rate in different dose of blocking and unblocking group.

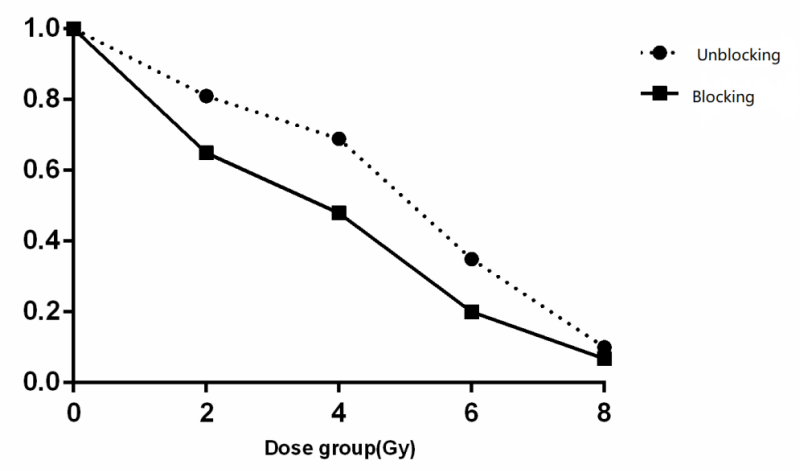

Figure 1. Cell survival rate in different dose of blocking and unblocking group.

\section{Apoptosis examination}

Spontaneous apoptosis existed in CNE cells. The apoptosis rate did not significantly increase after 2 Gy irradiation $(\mathrm{P}>0.05)$ before being blocked. The CNE cell significantly upregulated under the 2 Gy irradiation when the expression MAP2K6 was blocked $(\mathrm{P}<0.05)$.

\section{Discussion}

Previous study reported the proteins profiles based on the traditional Two-dimensional electrophoresis [13]. This method can usually identify about 1000 kinds of proteins, and conventional enzymolysis polypeptide based proteomics analysis method can identify about $3000-4000$ protein, which only cover the $5-20 \%$ of the whole proteins and is far from meet the requirement of high flux quantitative protein expression spectrum analysis. In the current study, we compared to proteins profiles from NPC sensitivity cells and NPC resistant cells through high throughput proteomics and found that MAP2K6 significantly expressed in human NPC radio resistant cells, which aims to provide new clues to reveal the molecular mechanism underlying NPC radio resistance.

The mitogen activated protein kinase (MAPK) includes extracellular regulated protein kinases, C-JUN N-terminal kinase, and p38 and ERK5 protein kinase. These proteins were important signal molecule. MAP3K6 was upstream kinase of p38 that was involved in cell proliferation, development and division. As a king of extracellular signal-regulated kinase, MAP2K was the integration points of multiple biochemical signaling pathways $[14,15]$. Under the effect of inflammatory cytokines and environmental stress, the MAP2K6 can encode 
proteins that exert phosphorylation and activation for p38MAPK mediated signal transduction pathways, including the suspension of cell cycle caused by environmental stress, transcriptional activation and apoptosis, and other intracellular reactions [16]. It was reported that $20 \%$ of mutations from promote the split the original activated protein kinase signaling pathways were associated with human tumors, and MAP2K6 plays an important role in human ovarian cancer metastasis $[17,18]$. It was reported that expression quantity of MAP2K6 in prostate cancer and esophagus, stomach and large intestine cancer were significantly raised compared with normal tissues [19]. We compared the expression of MAP2K6 in NPC cell radio sensitivity and resistance group. The expression of MAP2K6 in resistance group was significantly higher than that in the radio sensitivity group. We also compared the expression level of MAP2K6 in different dose level and found that the expression level of MAP2K6 increased by elevation of irradiation. We further test the results by inhibiting expression of MAP2K6 in two cells group. 2Gy irradiation was given to radio sensitivity and resistance groups. The cell survival rate of blocking group was significantly higher than that in the unblocking. This result proved that significant relation existed between expression of MAP2K6 and radio resistance. Our results indicated that expression of MAP2K6 can increase radio resistance of NPC CNE cells, and may could be a treatment target in NPC radiotherapy.

In conclusion, the high expression of MAP2K6 was associated with radio resistance in human NPC CNE cells. MAP2K6 may a potential biomarker of NPC radio sensitivity. The present study was limited in cell lines, and further research was required.

\section{References}

1. Han BL, Xu XY, Zhang CZ, Wu JJ, Han CF, Wang H, Wang X, Wang GS, Yang SJ, Xie Y. Systematic review on Epstein-Barr virus (EBV) DNA in diagnosis of nasopharyngeal carcinoma in Asian populations. Asian Pac J Cancer Prev 2012; 13: 2577-2581.

2. Chang ET, Adami HO. The enigmatic epidemiology of nasopharyngeal carcinoma. Cancer Epidemiol Biomarkers Prev 2006; 15:1765-1777.

3. Chua M, Wee J, Hui EP, Chan A. Nasopharyngeal carcinoma. Lancet 2016; 387:1012-1024.

4. Lee AW, Ma BB, Ng WT, Chan AT. Management of nasopharyngeal carcinoma: Current practice and future perspective. J Clin Oncol 2015; 33: 3356-3364.

5. Li Z, Li Y, Yan S, Fu J, Zhou Q, Huang X, Shen L. Nimotuzumab combined with concurrent chemoradiotherapy benefits patients with advanced nasopharyngeal carcinoma. Onco Targets Ther 2017; 10: 5445-5458.

6. Su SF, Han F, Zhao C, Chen CY, Xiao WW, Li JX, Lu TX. Long-term outcomes of early-stage nasopharyngeal carcinoma patients treated with intensity-modulated radiotherapy alone. Int $\mathrm{J}$ Radiat Oncol Biol Phys 2012; 82: 327-333.
7. Chen ZT, Liang ZG, Zhu XD. A Review: Proteomics in nasopharyngeal carcinoma. Int $\mathrm{J}$ Mol Sci 2015; 16: 15497-15530.

8. Qu C, Liang Z, Huang J, Zhao R, Su C, Wang S, Wang X, Zhang R, Lee MH, Yang H. MiR-205 determines the radioresistance of human nasopharyngeal carcinoma by directly targeting PTEN. Cell Cycle 2012; 11: 785-796.

9. Zhou Q, He Y, Zhao Y, Wang Y, Kuang W, Shen L. A Study of 358 Cases of locally advanced nasopharyngeal carcinoma receiving intensity-modulated radiation therapy: Improving the seventh edition of the American joint committee on cancer t-staging system. Biomed Res Int 2017; 2017: 1419676.

10. Li Z, Li Y, Xue J. Clinical significance of miRNA-433 expression in hepatocellular carcinoma. Biomed Res-India 2017; 28: 7661-7664.

11. Li ZZ, Shen LF, Li YY, Chen P, Chen LZ. Clinical utility of microRNA-378 as early diagnostic biomarker of human cancers: a meta-analysis of diagnostic test. Oncotarget 2016; 7: 58569-58578.

12. Li Z, Zhou Q, Li Y, Yan S, Fu J, Huang X, Shen L. Mean cerebral blood volume is an effective diagnostic index of recurrent and radiation injury in glioma patients: A metaanalysis of diagnostic test. Oncotarget 2017; 8: 15642-15650.

13. Feng XP, Yi H, Li MY, Li XH, Yi B, Zhang PF, Li C, Peng F, Tang CE, Li JL, Chen ZC, Xiao ZQ. Identification of biomarkers for predicting nasopharyngeal carcinoma response to radiotherapy by proteomics. Cancer Res 2010; 70: 3450-3462.

14. Davis RJ. Signal transduction by the JNK group of MAP kinases. Cell 2000; 103: 239-252.

15. Davis S, Laroche S. Mitogen-activated protein kinase/ extracellular regulated kinase signalling and memory stabilization: a review. Genes Brain Behav 2006; 2: 61-72.

16. Morozov A, Muzzio IA, Bourtchouladze R, Van-Strien N, Lapidus K, Yin D, Winder DG, Adams JP, Sweatt JD, Kandel ER. Rap1 couples cAMP signaling to a distinct pool of p42/44MAPK regulating excitability, synaptic plasticity, learning, and memory. Neuron 2003; 39: 309-325.

17. Hickson JA, Huo D, Vander GD, Lin A, Rinker-Schaeffer CW, Yamada SD. The p38 kinases MKK4 and MKK6 suppress metastatic colonization in human ovarian carcinoma. Cancer Res 2006; 66: 2264-2270.

18. Lotan TL, Lyon M, Huo D, Taxy JB, Brendler C, Foster BA, Stadler W, Rinker-Schaeffer CW. Up-regulation of MKK4, MKK6 and MKK7 during prostate cancer progression: an important role for SAPK signalling in prostatic neoplasia. J Pathol 2007; 212: 386-394.

19. Parray AA, Baba RA, Bhat HF, Wani L, Mokhdomi TA, Mushtaq U, Bhat SS, Kirmani D, Kuchay S, Wani MM, Khanday FA. MKK6 is upregulated in human esophageal, stomach, and colon cancers. Cancer Invest 2014; 32: 416-422. 


\section{*Correspondence to}

Zhanzhan Li

Department of Oncology

Xiangya Hospital

Central South University

PR China 